\title{
The effects of water-based exercise on postural control in children with spastic cerebral palsy
}

\author{
Kyung Ho Kim, Hwa Kyung Shin \\ Department of Physical Therapy, College of Bio and Medical Science, Daegu Catholic University, Gyeongsan, Republic of Korea
}

Objective: Swimming and water-based exercise (WE) programs can provide vigorous physical activity in a fun and a motivating environment. The properties of water, may make it easier for children with cerebral palsy (CP) to move and to participate in an WE program. The aims of this study was to evaluate the effect of WEs on postural control in children with spastic CP.

Design: Quasi-experimental design (one group pretest-posttest design).

Methods: Twenty preschoolers with spastic CP participated in this study. Prior to the application, sufficient warm-up training was performed to allow the subjects to adapt to the water. Afterwards, three different types of underwater leg exercises for the flexor, extensor, and adductor/abductor of muscles of the lower limb were performed. The WE program lasted during 8 weeks, with one-40 minute sessions per week ( 8 training session). The usual care was performed during the training session. The Korean-trunk control measurement scale (K-TCMS) and weight distribution variability (difference of both Fz) were measured before and after training session.

Results: According to the results, the K-TCMS scores of the three sub-levels, including static sitting, dynamic sitting, and dynamic reaching, was significantly increased after the training session $(p<0.05)$. Also, the weight distribution variability was significantly decreased after 8 weeks $(p<0.05)$.

Conclusions: We suggest that WEs may improve the postural control ability in children with spastic CP. Furthermore, we support the need for additional research on the effect of WE on gait or activity of daily living performance with a control group included.

Key Words: Cerebral palsy, Exercise, Posture, Water

\section{Introduction}

Cerebral palsy (CP), which is a non-progressive lesion in the developing fetus or neonatal brain, presents with a group of permanent impairments in the development of movements and postures due to immature brain lesions or disorders, leading to limitations in activity. Children with spastic CP present with difficulties in maintaining an anti-gravity posture due to lack of stability and mobility of the trunk, which in turn leads to difficulty in producing daily life movements because of an immature postural control mechanism.

One of the treatment methods for reducing muscle tension of children with $\mathrm{CP}$ is aquatic therapy exercise. Water-based exercise (WE) allows for a more effective exercise performance by lowering muscle tone through the relaxation effect of warm water, increasing pain threshold, and reducing muscle spasms. WE therapy also provides a safer and more efficient level of intervention due to the nature of the water compared to the ground-based exercise intervention method. All objects on the ground and underwater are affected by gravity. However, buoyancy reduces the effect of gravity, which reduces the load placed onto the joints and reduces the risk of joint damage. Buoyancy also assists the body of a child with muscle strength levels below the fair level, which

Received: 18 May, 2017 Revised: 14 June, 2017 Accepted: 14 June, 2017

Corresponding author: Hwa Kyung Shin

Department of Physical Therapy, College of Bio and Medical Science, Daegu Catholic University, 13-13 Hayang-ro, Geumnak-ri, Hayang-eup, Gyeongsan 38430, Republic of Korea

Tel: 82-53-850-2532 Fax: 82-53-359-6772 E-mail: hkshin1@cu.ac.kr

(c) This is an Open-Access article distributed under the terms of the Creative Commons Attribution Non-Commercial License (http://creativecommons.org/licens es/by-nc/4.0) which permits unrestricted non-commercial use, distribution, and reproduction in any medium, provided the original work is properly cited.

Copyright $@ 2017$ Korean Academy of Physical Therapy Rehabilitation Science 
makes it easier for the child to move. In addition, buoyancy helps maintain posture so that even if there is impaired motor function, it is easy to change posture or movements, and more joint and muscle movements can produce a positive effect on muscle tension [1].

In fact a study on the effect of the underwater rehabilitation program on motor ability in children with $\mathrm{CP}$ showed that WE therapy applied to infants 3 years of age showed a significant difference [2], and that aerobic exercises performed in the water had a positive effect on muscle strength and balance ability, gait pattern and energy consumption efficiency in 5-year-old children with bilateral paralysis [3].

Currently, various exercise programs have been developed and implemented to improve the ability to control the posture of children with CP [4]. However, most of these exercises are performed on the ground rather than in the water. Therefore, the purpose of this study is to investigate the effects of aquatic therapy exercises on postural control ability in paralyzed children.

\section{Methods}

\section{Subjects}

Twenty children (13 males, 7 females) who were diagnosed with spastic CP participated in this study. The selection criteria of the study subjects are as follows: children who could walk, could understand the procedure, did not have swallowing difficulties and are not afraid of being in water, had no congenital or neurological disorders other than $\mathrm{CP}$, had no open wound or active infection, and did not undergo surgical operation or Botox treatment within the last 6 months.

The subjects agreed to participate in the study and pro- vided their informed consent after being fulling explained of the procedure. The study was approved by the Institutional Review Board of the College of Bio and Medical Science, Daegu Catholic University (IRB No. CUIRB-2016-0108). The mean age, height, and weight of the subjects were $5.0 \pm 2.0,99.5 \pm 20.7$, and $16.65 \pm 0.15$ respectively.

\section{Research design}

The WEs were performed after the subjects had undergone sufficient amount of water adaptation training. The water exercises were performed once a week for 40 minutes for 8 weeks. To assess postural control ability before and after intervention, the Korean-trunk control measurement scale (K-TCMS) and the weight distribution variability was used as outcome measures.

\section{Water-based exercise}

Flexion exercise consisted of simultaneous flexion and crossed flexion (Figure 1). The simultaneous flexion of was carried out by having the therapist holding onto the child's waist and the child facing the front with both knees bent and touching the knees for 5 seconds. crossed flexion was carried out with alternately on one leg by same method.

The resisted extension exercise consisted of resisted extension of single limb, non-resisted extension of single limb, and resisted crossed extension (Figure 2). For of resisted extension of lower limb, the child held onto the bar in prone position in a hip extension and knee flexion position and the therapist provided resistance onto the foot of the subject against knee extension for 5 seconds in order to maintain knee flexion. For the non-resisted extension of single limb, the child held onto the bar in prone position in hip and knee extension position and lifted the lower extremity. For re-
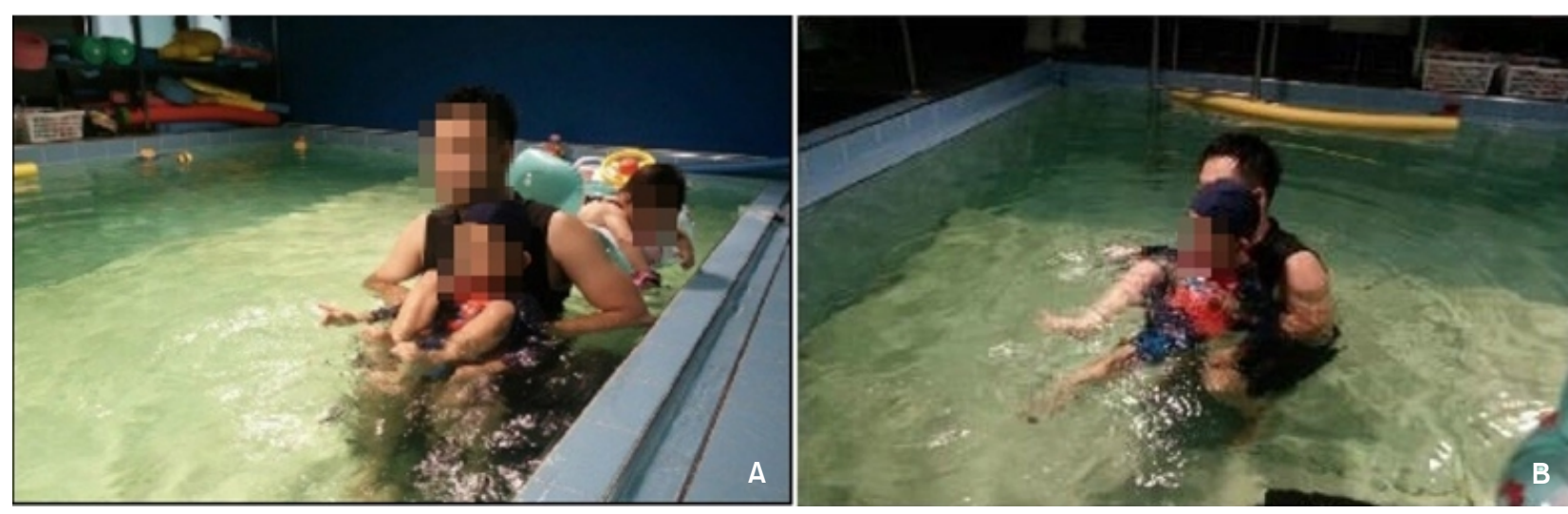

Figure 1. Flexion exercise of lower limb. (A) Simultaneous flexion. (B) Crossed flexion. 

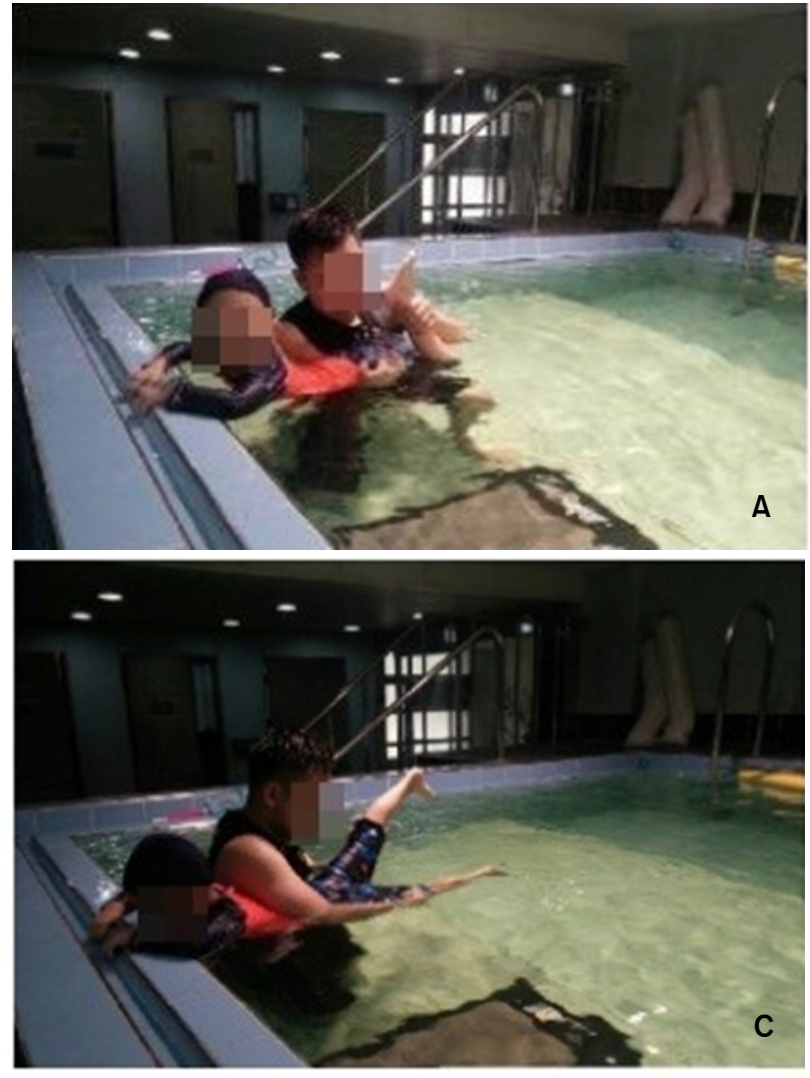

sisted crossed extension of single limb, the child held onto the bar in prone position in hip and knee extension position and lifted the lower extremity alternately.

Abduction and adduction exercise consisted of unilateral adduction and abduction, bilateral adduction and abduction (Figure 3) $[5,6]$. For the single lower extremity horizontal adduction exercise, the child held onto the bar in prone position and with bilateral knee extended and after placing one lower extremity in abduction, the therapist provided resistance for 5 seconds while the child brought the abducted lower extremity into adduction. For the single lower extremity horizontal abduction exercise, the child held onto the bar in prone position and with bilateral knee extended and while the child was performing a single lower extremity abduction movement, the therapist provided resistance against the heel for 5 seconds. For the bilateral lower extremity horizontal abduction exercise, the child held onto the bar in prone position and with bilateral knee extended and while the child was performing bilateral lower extremity abduction movement, the therapist provided resistance against both heels for 5 seconds.

Each exercise was performed for 3 sets where 1 set equaled 10 repetitions. Subjects were provided a rest inter-

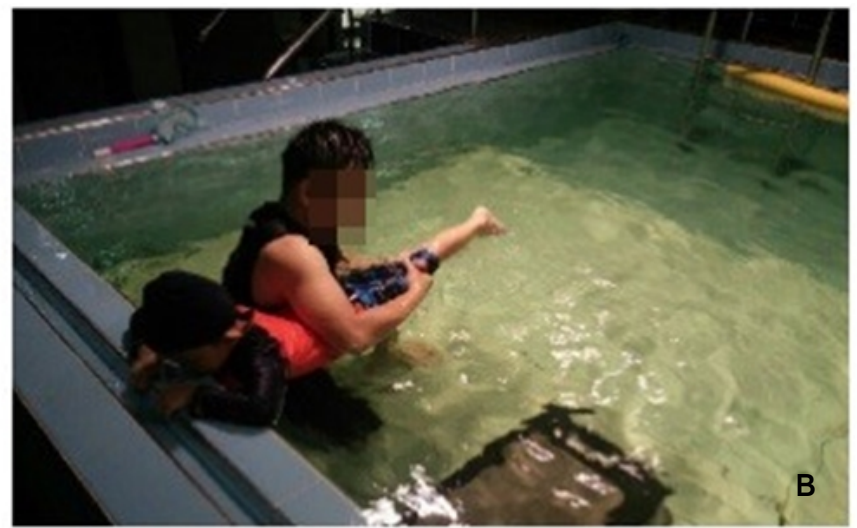

Figure 2. Resisted extension of lower limb. (A) Resisted extension of one limb. (B) Non-resisted extension of one limb. (C) Resisted cross extension.

val of 3 minutes between each set.

\section{Research equipment and measuring tools}

\section{Korean-trunk control measurement scale}

The K-TCMS was used to measure the key components of functional regulation of $\mathrm{CP}$. The starting posture for each exercise was the same without the application of any devices on the foot or trunk. The assessment began with a child sitting on a treatment table without a backrest. The arm and leg rests were not used, and the entire thigh was in contact with the tabletop. The subject's hands were kept near the trunk and at the start signal of the instructor, the subject was instructed to sit in an upright position and to maintain that position during the time of experiment [7]. Proper posture was considered as the most upright posture the child could maintain and varied from child to child. The postures served as a reference for measuring the degree of deviation from the starting posture when performing the movement. Each item was measured three times and the best score was used. If a child required support of one arm while performing a subset of the static sitting balance, the child was asked to place the upper extremities extended on the table.

The K-TCMS consists of 15 items, with 5 static balance 

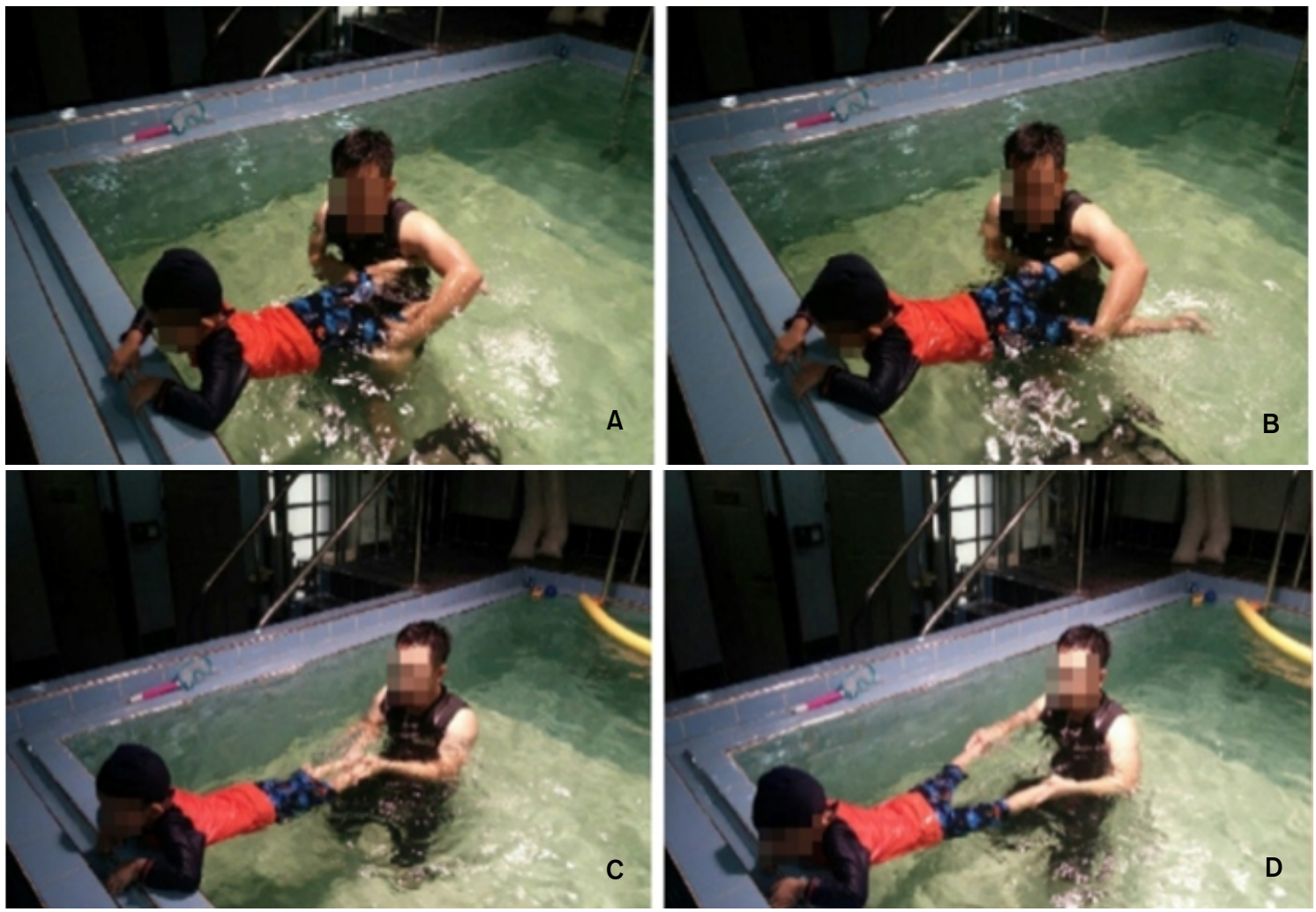

Figure 3. Horizontal adduction and abduction movement of lower limb. (A) Unilateral adduction. (B) Unilateral abduction. (C) Bilateral adduction. (D) Bilateral abduction.

items, 7 dynamic balance items, and 3 dynamic reach items in the sitting position, totaling up to 58 points. Higher scores indicate better trunk control ability [8]. The subscale score of the TCMS has a very good test-retest intra-class correlation coefficient (ICC) score of 0.97 and an inter-test ICC score of $0.98[9,10]$.

\section{Weight distribution variability}

A force plate (Advanced Mechanical Technology Inc. [AMTI], Watertown, MA, USA) was used measure and collect data for variability of body weight distribution. The plate was preheated for about 45 minutes to remove the electronic drift and was then used for the experiment. For this study, two force plates were used to measure the change in the movement path of the vertical ground reaction force center of pressure. Prior to the experiment, after calibration to zero, the feet were placed on the floor plate and the assessment was performed in a standing position. The difference between the two $\mathrm{Fz}$ values was defined as a representative value.

\section{Statistical analysis}

The data collected through this study were compared with the mean and standard deviation of each variable using IBM SPSS Statistics ver. 22.0 (IBM Co., Armonk, NY, USA). The between-group differences were analyzed using pretreatment TCMS scores and pre-treatment post-treatment weight changes were analyzed using the paired t-test. The statistical significance level was set as $\alpha=0.05$.

\section{Results}

KTCMS of the children with spastic $\mathrm{CP}$ was significantly higher in static sitting balance, dynamic sitting balance, and dynamic reach after WE $(p<0.05$; Table 1$)$. Fz difference of both side for weight distribution variability was significantly lower after WE $(p<0.05$; Table 1$)$.

\section{Discussion}

Decreased muscle tone in children with $\mathrm{CP}$ has important implications for treatment of $\mathrm{CP}$ and among them, exercises 
Table 1. Change of K-TCMS

\begin{tabular}{lcccc}
\hline \multicolumn{1}{c}{ K-TCMS } & Pre-test & Post-test & t & $p$ \\
\hline Static sitting balance & $17.15(1.81)$ & $18.10(1.48)$ & -6.89 & $0.00^{*}$ \\
Dynamic sitting balance & $15.25(3.44)$ & $16.95(3.85)$ & -8.79 & $0.00^{*}$ \\
Dynamic reaching & $8.50(0.60)$ & $9.75(0.63)$ & -8.75 & $0.00^{*}$ \\
Weight distribution variability & $95.54(22.15)$ & $93.79(21.62)$ & 4.05 & $0.00^{*}$ \\
\hline
\end{tabular}

Values are presented as mean (SD).

K-TCMS: Korean-trunk control measurement scale. ${ }^{*} p<0.05$.

that can reduce muscle tension by using the buoyancy of water can be used as part of an underwater rehabilitation program. Among the many interventions for children with $\mathrm{CP}$ with abnormal gait patterns, WEs are performed in various ways, such as in the pool or in the tank and that it is a way to promote the application of various therapeutic interventions, including muscle strengthening, joint mobilization, balance, gait and endurance training. Underwater motion, which produces resistance in all active movements, utlizes the physical properties of water that directly affect the body, such as buoyancy, hydrostatic pressure, and resistance to water [11]. Buoyancy is the force of the same amount of liquid that pushes the immersed body against gravity upwares, which reduces the load on the joint due to weight and allows three-dimensional motion. Buoyancy also allows one to succeed in performing exercises that would be difficult to perform on ground and assists to maintain posture so that even with impaired exercise function, it allows one to change positions and perform an exercise pattern easily and also reduces muscle tension and the load placed onto the joints. In addition, warm water can decrease muscle tension, muscle spasm, and relieve muscle tension, which can promote a more effective way to exercise. Also, increased muscle strength can be expected by increasing the speed of movement in the opposite direction fo the flow of water [12].

The results of this study showed that after intervention, the trunk control scale was significantly higher in static balance, dynamic balance, and dynamic reach, and variability in weight distribution was significantly lower compared to before intervention. This indicates that WE can improve the postural control function of children with spastic CP. This study can be distinguished from the existing studies in that bilateral lower extremity exercises that included flexion, extension, adduction and abduction were included order to improve postural control function of children with spastic CP.

The results of this study suggest that the effect of the low- er extremity strength training performed underwater improved the postural control function of children with $\mathrm{CP}$. During the study, children with CP who were not able to move smoothly due to gravity and the physical limitations of the ground seemed to be able to actively and pleasantly perform the WEs. Therefore, it is considered that WE programs for children with CP may be used to motivate children who lack confidence in exercising on the ground. Movement in the water is free from the risk of falling compared to the ground, and because it is free and comfortable, abnormal tone can be stabilized, which allows for the children with CP to actively participate in the exercise program and exhibit improved postural control function [13-17].

Ballaz et al. [5] reported improvement instanding and walking ability of children with CP with low physical abilities with a Gross Motor Function Classification System score of 3 to 4 . This is consistent with the results of this study. This is due to the fact that WE programs reduce muscle tension in children with $\mathrm{CP}$ and improve balance ability as a result of psychological stabilityand buoyancy. It is considered that it is possible to improve not only trunk control ability but also balance ability of the lower extremity through lower extremity exercises performed with decreased body tension. In this study, only 20 children with CP were included. Therefore, a comparative study with a ground intervention program with a control group included will be needed in the future.

In order to improve postural control ability in children with spastic CP, an WE program was proposed and the effects of WE on postural control were examined. The results of this study showed that after intervention, the trunk control scale was significantly higher in both static balance, dynamic balance, and dynamic reach compared to before intervention. WEs can improve postural control function of children with spastic $\mathrm{CP}$. 


\section{Acknowledgements}

This work was supported by research grants from the Daegu Catholic University in 2015.

\section{Conflict of Interest}

The authors declared no potential conflicts of interest with respect to the authorship and/or publication of this article.

\section{References}

1. Rose J, Wolff DR, Jones VK, Bloch DA, Oehlert JW, Gamble JG. Postural balance in children with cerebral palsy. Dev Med Child Neurol 2002;44:58-63.

2. Salem Y, Gropack SJ. Aquatic therapy for a child with type III spinal muscular atrophy: a case report. Phys Occup Ther Pediatr 2010;30:313-24.

3. Retarekar R, Fragala-Pinkham MA, Townsend EL. Effects of aquatic aerobic exercise for a child with cerebral palsy: single-subject design. Pediatr Phys Ther 2009;21:336-44.

4. Gorter JW, Currie SJ. Aquatic exercise programs for children and adolescents with cerebral palsy: what do we know and where do we go? Int J Pediatr 2011;2011:712165.

5. Ballaz L, Plamondon S, Lemay M. Group aquatic training improves gait efficiency in adolescents with cerebral palsy. Disabil Rehabil 2011;33:1616-24

6. Kelly M, Darrah J. Aquatic exercise for children with cerebral palsy. Dev Med Child Neurol 2005;47:838-42.

7. Pham HP, Eidem A, Hansen G, Nyquist A, Vik T, Sæther R. Validity and responsiveness of the trunk impairment scale and trunk control measurement scale in young individuals with cere- bral palsy. Phys Occup Ther Pediatr 2016;36:440-52.

8. Mitteregger E, Marsico P, Balzer J, van Hedel HJ. Translation and construct validity of the Trunk Control Measurement Scale in children and youths with brain lesions. Res Dev Disabil 2015; 45-46:343-52.

9. Sæther R, Jørgensen L. Intra- and inter-observer reliability of the trunk impairment scale for children with cerebral palsy. Res Dev Disabil 2011;32:727-39.

10. Sæther R, Helbostad JL, Adde L, Braendvik S, Lydersen S, Vik $\mathrm{T}$. The relationship between trunk control in sitting and during gait in children and adolescents with cerebral palsy. Dev Med Child Neurol 2015;57:344-50.

11. Fragala-Pinkham MA, Smith HJ, Lombard KA, Barlow C, O'Neil ME. Aquatic aerobic exercise for children with cerebral palsy: a pilot intervention study. Physiother Theory Pract 2014; 30:69-78.

12. Dimitrijević L, Bjelaković B, Lazović M, Stanković I, Čolović $\mathrm{H}$, Kocić $\mathrm{M}$, et al. [Aquatic exercise in the treatment of children with cerebral palsy]. Srp Arh Celok Lek 2012;140:746-50. Serbian.

13. Kenis-Coskun O, Giray E, Eren B, Ozkok O, Karadag-Saygi E. Evaluation of postural stability in children with hemiplegic cerebral palsy. J Phys Ther Sci 2016;28:1398-402.

14. Han HK, Chung YJ. Effects of task-oriented training for gross motor function measure, balance and gait function in persons with cerebral palsy. Phys Ther Rehabil Sci 2016;5:9-14.

15. Yun CK. Relationship between the quality of life of the caregiver and motor function of children with cerebral palsy. Phys Ther Rehabil Sci 2017;6:26-32.

16. Lee JW, Hwang SJ. Effects of balance imagery of semi-tandem stance on a flat floor and balance beam for postural control: a comparison between older and younger adults. Phys Ther Rehabil Sci 2015;4:87-93.

17. Jung JH, Ko SE, Lee SW. Immediate effects of single-leg stance exercise on dynamic balance, weight bearing and gait cycle in stroke patients. Phys Ther Rehabil Sci 2014;3:49-54. 